\title{
A multi-wavelength view of the Galactic center dust ridge reveals little star formation
}

\author{
K. Immer ${ }^{1,2}$, K. M. Menten ${ }^{1}$, F. Schuller ${ }^{3,1}$, and D. C. Lis ${ }^{4}$ \\ 1 Max-Planck-Institut für Radioastronomie, Auf dem Hügel 69, 53121 Bonn, Germany \\ e-mail: kimmer@mpifr-bonn.mpg.de \\ 2 Harvard-Smithsonian Center for Astrophysics, 60 Garden Street, 02138 Cambridge, MA, USA \\ 3 ESO, Alonso de Cordova 3107, Casilla 19001, Santiago 19, Chile \\ 4 California Institute of Technology, Cahill Center for Astronomy and Astrophysics 301-17, Pasadena, CA 91125, USA
}

Received 7 March 2012 / Accepted 1 November 2012

\begin{abstract}
The Galactic center dust ridge consists of a narrow string of massive condensations, first identified in submillimeter dust continuum emission. To determine whether new high-mass stars are forming in this region, we performed new observations at $870 \mu \mathrm{m}$ with the Atacama Pathfinder EXperiment (APEX) telescope and at $8.4 \mathrm{GHz}$ with the Very Large Array. We complement our data with recent maser and mid-infrared results. The ridge's clouds are dark at mid-infrared wavelengths, indicating the presence of cold, high column density material. In combination with existing temperature measurements in the dust ridge, we determine masses of the largest clouds. The results show that the dust ridge contains a very massive reservoir of molecular material. We find five radio continuum sources at $8.4 \mathrm{GHz}$ in the general dust ridge vicinity, but outside of the dust ridge clouds. They are likely all excited by massive young stars, whose properties we constrain. Our observations exclude the existence of zero age main sequence stars with spectral types earlier than B0.5 within the dust ridge clouds. The only indication of ongoing high-mass star formation inside the clouds are class II methanol masers that are found in two of the clouds. Except for a weak water maser, found in previous observations, no signs of star formation are detected in the most massive cloud, M0.25+0.012.
\end{abstract}

Key words. Galaxy: center - stars: formation - H II regions

\section{Introduction}

The dust ridge (named by Lis \& Carlstrom 1994) is an accumulation of clumpy dust condensations near the Galactic center, which are located along an arc-like narrow ridge, connecting the radio continuum sources G0.18-0.04 and Sgr B1 at Galactic longitude $l \approx 0.5$.

To determine the dust temperature of the molecular clouds, Lis \& Menten (1998) and Lis et al. (1999) imaged the region using the Long Wavelength Spectrometer (LWS) aboard the Infrared Space Observatory (ISO) at wavelengths from 45 to $175 \mu \mathrm{m}$. They found that the distribution of the emission at wavelengths longer than $70 \mu \mathrm{m}$ is well correlated with the submillimeter continuum emission whereas the clouds are seen in absorption against the general Galactic center background at shorter wavelengths. This implies that the clouds are much colder than and located in front of the warm dust responsible for the emission at $70 \mu \mathrm{m}$ and that they must have a high continuum opacity at $70 \mu \mathrm{m}$. In addition, Lis et al. (1999) estimated the temperature at six locations by fitting the observed spectral energy distributions (SEDs) with a two-component grey-body model. They found out that the bulk of the dust has temperatures between 13 and $20 \mathrm{~K}$, but that a small amount of warmer dust is also present, which potentially corresponds to emission from externally heated cloud surfaces. Ammonia observations of M0.25+0.012 by Güsten et al. (1981) suggest that the gaseous material has higher temperatures than the dust, probably being heated by cosmic rays.

Molinari et al. (2011) interpret the dust ridge as a quarter of a $100 \mathrm{pc}$ size elliptical twisted ring of molecular material around the Galactic center. Their Herschel observations of the central molecular zone show the dust ridge in absorption at $70 \mu \mathrm{m}$ and in emission at $250 \mu \mathrm{m}$, confirming the low temperatures of the dust ridge clouds, estimated by Lis et al. (1999). The column density map of atomic hydrogen, derived from the $250 \mu \mathrm{m}$ map, shows strong peaks at the position of the dust ridge clouds, indicating high column densities.

Lis \& Menten (1998) determined the distribution of the $\mathrm{CO}(2-1)$ and the $\mathrm{HCO}^{+}(3-2)$ emission at the location of $\mathrm{M} 0.25+0.012$, the largest cloud in the dust ridge. The line widths of these molecules are very broad $\left(\sim 30 \mathrm{~km} \mathrm{~s}^{-1}\right.$ FWHM), similar to typical line widths observed in giant molecular clouds (GMCs) in the Galactic center, indicating that M0.25+0.012 is likely located in the Galactic center region (i.e., at $8.5 \mathrm{kpc}$, Ghez et al. 2008).

Furthermore, Lis et al. detected a large velocity gradient across the southern part of the cloud indicating streaming gas motions or several spatially overlapping velocity components. This could be a sign of a cloud collision taking place. As the cloud has not yet been warmed up by shocks, it is likely that the collision is at an early stage and may trigger substantial highmass star formation inside the cloud in the future.

M0.25+0.012 was recently studied by Longmore et al. (2012), who present data from near-infrared to millimeter wavelengths. They determined global properties such as dust mass, dust temperature, and column density for this cloud and estimated the distance to the cloud from extinction measurements. Their results are consisted with the Galactic center distance of $8.5 \mathrm{kpc}$ and the temperature measurements of Lis et al. (1999). 
Table 1. Observation parameters at $8.4 \mathrm{GHz}$.

\begin{tabular}{lccc}
\hline \hline Target name & $\begin{array}{c}\text { Total on-source } \\
\text { time } \\
{[\mathrm{s}]}\end{array}$ & $\begin{array}{c}\text { Pointing center } \\
\text { RA (J2000) } \\
\text { [hh mm ss.ss] }\end{array}$ & $\begin{array}{c}\text { Dec (J2000) } \\
{\left[\mathrm{dd}^{\prime \prime} \text { '"' }^{\prime \prime \prime \prime \prime}\right]}\end{array}$ \\
\hline B1328+307 & 200 & 133108.29 & +303033.04 \\
B1730-130 & 1820 & 173302.69 & -130449.55 \\
POS1 & 3720 & 174608.59 & -284237.49 \\
POS2 & 4180 & 174610.17 & -283807.37 \\
POS3 & 4640 & 174622.09 & -283436.50 \\
\hline
\end{tabular}

They conclude that this cloud could be a precursor to an Arches-like young massive cluster.

In this paper, we present new observations of the dust ridge at $870 \mu \mathrm{m}$ with the Atacama Pathfinder EXperiment (APEX) telescope and at $8.4 \mathrm{GHz}$ with the Very Large Array (VLA). In Sect. 2, we describe the observations and the data reduction of the two data sets. Section 3 gives a comprehensive picture of the dust ridge at infrared, submillimeter, and radio wavelengths. In the last section, we summarize our results.

\section{Observation and data reduction}

Three target positions (POS1, POS2, POS3) were observed in 1993 June at $8.4 \mathrm{GHz}$ in the BC hybrid configuration with the NRAO Very Large Array (VLA) covering almost the whole dust ridge. Data were recorded in two intermediate frequency (IF) bands with opposite (right and left) circular polarization, centered at $8.4149 \mathrm{GHz}(\mathrm{IF} 1=\mathrm{RCP})$ and $8.4649 \mathrm{GHz}(\mathrm{IF} 2=\mathrm{LCP})$. Each band was $50 \mathrm{MHz}$ wide (46 MHz effective bandwidth).

Fourteen scans on the phase calibrator B1730-130 were recorded before, as well as after the observations of the target sources, resulting in a total on-source time of half an hour. B1328+30, the amplitude calibrator, was observed only once for $200 \mathrm{~s}$. The total integration time of each target source, comprising 12 or 13 individual scans, is larger than one hour. The exact values are listed together with the pointing centers of the observed fields in Table 1 (Cols. 2-4; Col. 1 gives the target names).

After "flagging" time ranges in which certain baselines contained obviously bad data and the amplitude and phase calibration, several self-calibration cycles were repeated and the final images, restored with an elliptical Gaussian beam of $\sim 3{ }^{\prime \prime} 6 \times \sim 2$ '. $5 F W H M$, were then corrected for primary beam attenuation. At our observing frequency, the primary beam has a FWHM size of 5!4, which determines the field of view. The uncertainty of the absolute flux calibration at $8.4 \mathrm{GHz}$ is less than $10 \%$, as determined from observations of the quasar 1730-130.

The images resulting from the POS1, POS2, and POS 3 pointings have rms values of $0.25,0.15$, and $0.25 \mathrm{mJy}^{\text {beam }}{ }^{-1}$. We will compare our radio observations with a VLA map, taken at $1.4 \mathrm{GHz}$ by Yusef-Zadeh et al. (2004) with a $1-\sigma$-sensitivity of $\sim 12 \mathrm{mJy} \mathrm{beam}^{-1}$ (in the dust ridge region) in a $\sim 30^{\prime \prime}$ beam, and a VLA catalog of discrete sources at 5 and $1.4 \mathrm{GHz}$ from Becker et al. (1994). Sources in this catalog are detected down to $9 \mathrm{mJy}$ at $5 \mathrm{GHz}$ and $10 \mathrm{mJy}$ at $1.4 \mathrm{GHz}$ with a resolution of $4-5^{\prime \prime}$. Our VLA observations have higher resolution and are a factor of 10-40 more sensitive than these comparable observations.

The submillimeter images of the dust ridge at $870 \mu \mathrm{m}$ were taken in the framework of the ATLASGAL survey
(Schuller et al. 2009) with the APEX ${ }^{1}$ telescope (Güsten et al. 2006). The resolution of the submillimeter maps is $19 ! 2$, and the map has been sampled on $2^{\prime \prime}$ pixels. The rms between pixels is in the range $40-60 \mathrm{mJy}^{\text {beam }}{ }^{-1}$ in regions with no emission. As compared to the data presented in Schuller et al. (2009), more coverages of the full region have been combined to produce the map that we use in the present paper. The field was covered by combining eight on-the-fly maps, each roughly orthogonal to the Galactic plane. Each map was executed five times with slightly changing orientation to minimize striping effects. Also, we used a slightly different reduction pipeline, specially developed in order to recover as much extended emission as possible. The main idea is to build the map in an iterative way, starting with the assumption that there is no emission on the edges of the map (at $b= \pm 1$ ). Then, the processing is similar to the one described in Schuller et al. (2009), but a major difference concerns the correlated noise removal. As in the original pipeline, a median value of all bolometer signals is computed at each integration, and is considered as sky signal, $S$, to be subtracted from all signals. But, in addition, the bolometer where the signal is equal to the median value, $S$, is identified. The position observed by this bolometer at this time is then uniquely determined. The procedure then looks for the intensity at this position in the map used as input model (i.e. the map resulting from the previous iteration). If there is significant emission, $E$, then $S$ is not subtracted from all signals, but only the difference $S-E$ is considered a sky signal and subtracted. This way, real astronomical emission is preserved from one iteration to the next.

We also made use of Spitzer/IRAC images at 3.6, 4.5, 5.8 , and $8.0 \mu \mathrm{m}$ that were obtained as part of the Galactic Legacy Infrared Mid-Plane Survey Extraordinaire (GLIMPSE) (Churchwell et al. 2009).

\section{High-mass star formation in the dust ridge}

\subsection{Dust emission in the dust ridge}

Figure 1 presents an image of the $870 \mu \mathrm{m}$ emission from the central molecular zone $(|l|<1.5,|b|<0.5)$. The location of the dust ridge is indicated, as well as the famous molecular complexes Sgr A, Sgr B1, Sgr B2, Sgr C, and Sgr D. From multi-wavelength submillimeter observations one concludes that most of this emission is produced by dust associated with these complexes.

Despite the better sensitivity of the Herschel Hi-GAL (Molinari et al. 2010) project, a comparison of our Fig. 1 with Herschel observations at $250 \mu \mathrm{m}$ (see Fig. 2 in Molinari et al. 2011) shows a very similar distribution of the dust emission. The Hi-GAL observations cover a wavelength range from 70 to $500 \mu \mathrm{m}$, showing dust of higher temperature than our ATLASGAL observations. Since the Hi-GAL data and the ATLASGAL observations have comparable resolutions, they can be used in combination to derive spectral energy distributions, and thus the temperatures, of the molecular clouds.

Figure 2 shows the $870 \mu \mathrm{m}$ emission of the dust ridge (red contour). The ridge consists of several dust condensations and extends, from Sagittarius B1 to M0.25+0.012, over $0.25^{\circ}$ (40 pc at the Galactic center distance of $8.5 \mathrm{kpc}$ ). Several weaker dust condensations are detected in the south of the dust ridge which form a projected ellipse with the dust ridge clouds. The letters indicate the condensations identified by Lis et al. (1999), for

\footnotetext{
1 The APEX project is a collaborative effort between the Max Planck Institute for Radioastronomy, the Onsala Space Observatory, and the European Southern Observatory.
} 


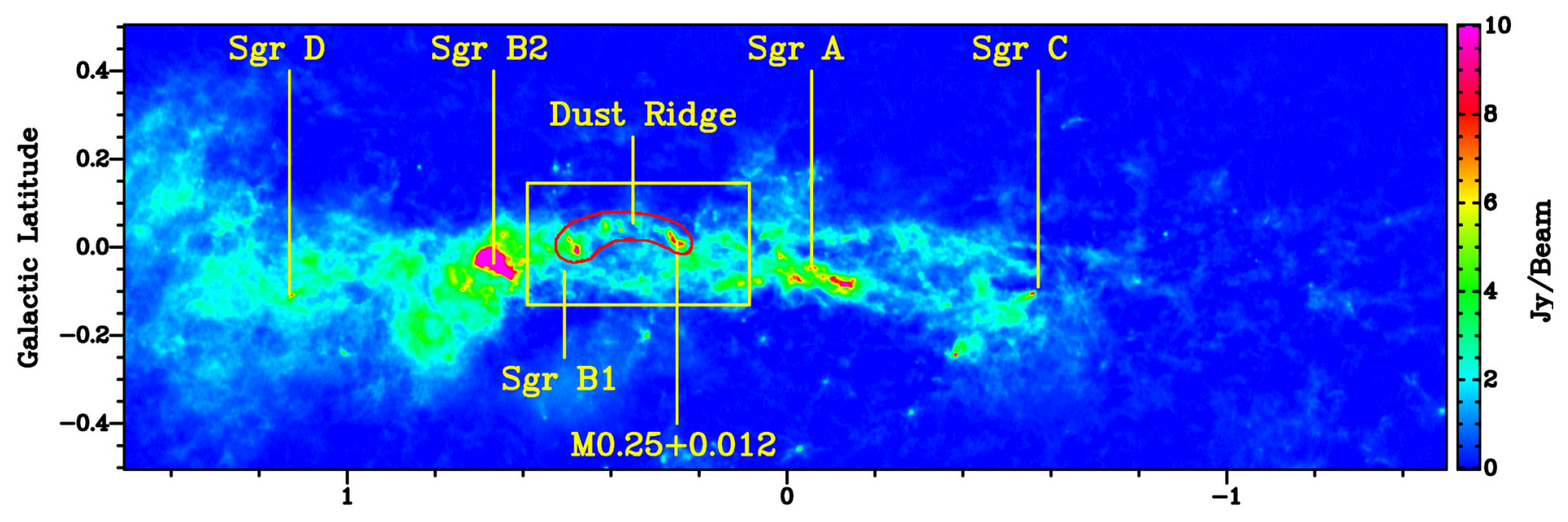

Galactic Longitude

Fig. 1. $870 \mu \mathrm{m}$ dust continuum emission of the central molecular zone. The figure shows the position of the dust ridge (red contour) and the large molecular cloud M0.25+0.012 in relation to other famous molecular complexes: Sgr A, Sgr B1, Sgr B2, Sgr C, and Sgr D. The region within the yellow box is enlarged in Fig. 2.

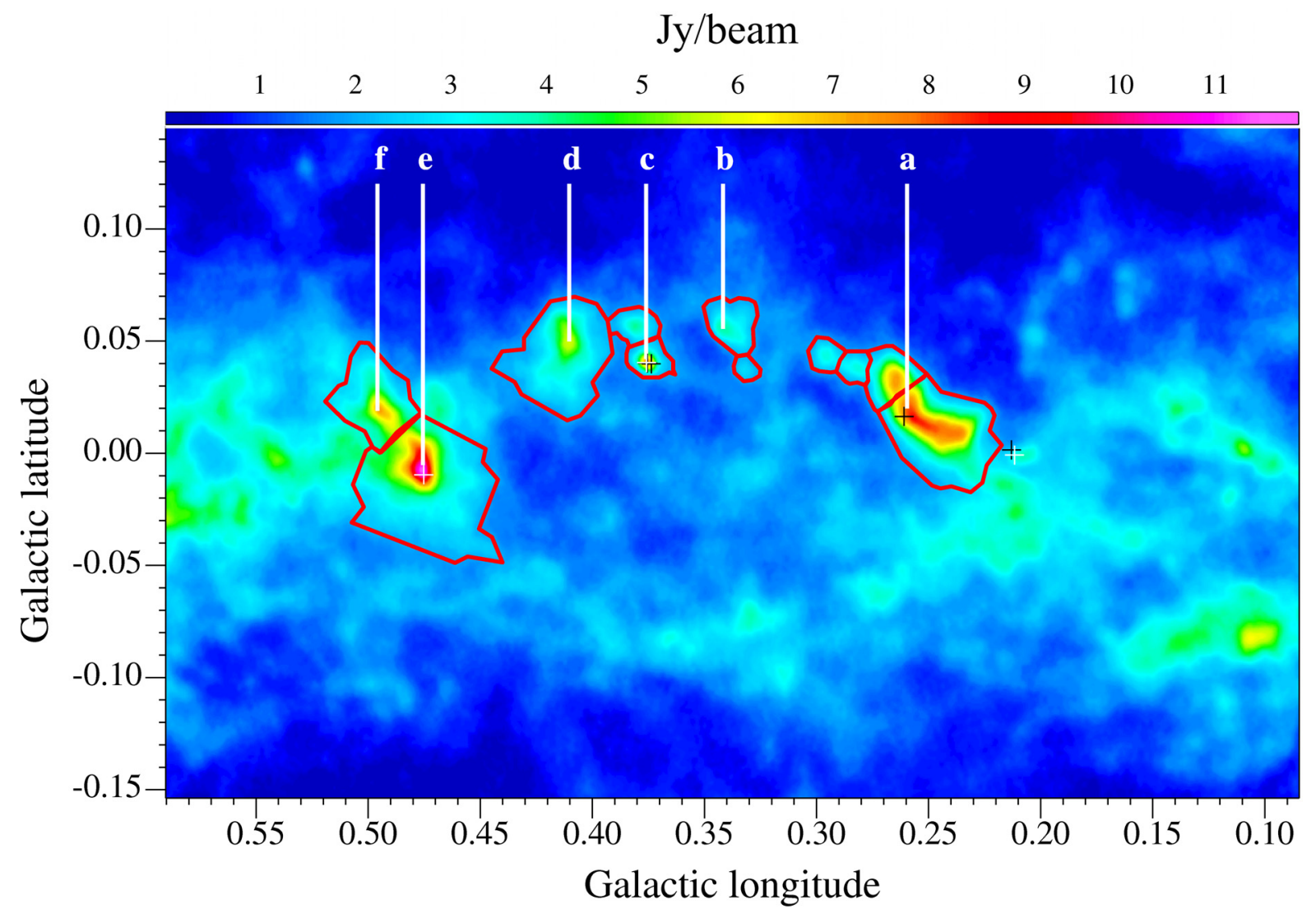

Fig. 2. Dust continuum emission towards the dust ridge (arc of dust condensations bent to the north) at $870 \mu$ m. The contours show the sizes of the molecular clouds over which the emission was integrated for the cloud mass determination. Water and methanol masers are marked with black and white crosses, respectively. The letters mark the positions at which Lis et al. (1999) determined the dust temperature from the SED fits.

which dust temperatures were determined from the ISO data. The lower longitude end of the ridge is located at a projected distance of around $30 \mathrm{pc}$ from the Galactic center. Figure 2 shows that the $870 \mu \mathrm{m}$ appearance of the dust ridge region is dominated by diffuse emission with a mean value of $\sim 1.7 \mathrm{Jy} \mathrm{beam}^{-1}$. To separate the diffuse emission from the emission of core regions and identify clumps of emission, we used the CUPID package of the starlink software with the established ClumpFind algorithm (Williams et al. 1994). We chose $1.7 \mathrm{Jy} \mathrm{beam}^{-1}$ as the lowest contour level and $0.3 \mathrm{Jy}^{\text {beam }}{ }^{-1}(5 \sigma)$ as the gap between contour levels. The impact of choosing different ClumpFind levels is discussed later in the text. The red contours in Fig. 2 show the boundaries of the identified clumps. Clouds a, b, and c are subdivided in several clumps. To quantify the cloud sizes, we assigned equivalent diameters based on the clump areas given by the ClumpFind algorithm.

To determine if the dust ridge clouds are gravitationally bound, the total masses of the clouds from molecular gas and dust and their virial masses have to be compared. The virial mass of a cloud is proportional to the radius of the cloud and 
Table 2. Temperatures and masses of sub-condensations in the dust ridge in the nomenclature of Lis et al. (1999).

\begin{tabular}{lcccc}
\hline \hline Position $^{a}$ & $T_{\text {Dust }}[\mathrm{K}]$ & $F_{870 \mu \mathrm{m}, \text { Int. }}[\mathrm{Jy}]$ & $M_{\text {Cloud }}\left[10^{3} M_{\odot}\right]$ & $D_{\text {Cloud }}\left[{ }^{\prime \prime}\right]$ \\
\hline $\mathrm{a}^{b}$ & 18 & 310 & 141 & 207 \\
$\mathrm{~b}$ & 22 & 39 & 13 & 96 \\
$\mathrm{c}$ & 20 & 46 & 18 & 96 \\
$\mathrm{~d}$ & 17 & 145 & 72 & 171 \\
$\mathrm{e}$ & 17 & 307 & 153 & 220 \\
$\mathrm{f}$ & 15 & 118 & 72 & 133 \\
\hline
\end{tabular}

Notes. ${ }^{(a)}$ see also Fig. 2. ${ }^{(b)} \mathrm{M} 0.25+0.012$.

the linewidth of the molecular emission squared. However, our submillimeter observations do not provide velocity information, thus prohibiting the determination of the virial mass. In a following paper, we study the kinematics of the cloud gas using data from the Millimetre Astronomy Legacy Team $90 \mathrm{GHz}$ Survey (MALT90, Foster et al. 2011) and recent APEX spectral line observations to investigate whether the clouds are gravitationally bound. Longmore et al. (2012) showed that M0.25+0.012 (cloud a) is indeed gravitationally bound. In this paper, we can only assume that the other clouds are also gravitationally bound and not only line-of-sight superpositions. Assuming further that the dust temperature, $T_{\mathrm{D}}$, does not change significantly within each cloud, we can determine the projected masses of the clouds $M_{\text {Cloud }}$ with the formula (following Hildebrand 1983)

$M_{\text {Cloud }}=\frac{d^{2} F_{870 \mu \mathrm{m}, \text { Int }} R}{B_{870 \mu \mathrm{m}}\left(T_{\mathrm{D}}\right) \kappa_{870 \mu \mathrm{m}}}$.

The integrated flux densities $F_{870 \mu \text { m,Int }}$ of the six clouds are measured by adding up all pixel flux density values within the boundaries of each clump. For the clouds a, b, and c, we summed up the integrated flux densities of all clumps belonging to the same cloud. For the gas-to-dust ratio, $R$, the opacity at $870 \mu \mathrm{m} \kappa 870 \mu \mathrm{m}$, and the distance to the clouds, $d$, values of $100,0.185 \mathrm{~m}^{2} \mathrm{~kg}^{-1}$, and $8.5 \mathrm{kpc}$, respectively, are assumed. The results are shown in Table 2 with Cols. 2 and 3 giving the dust temperatures and the integrated flux density values at $870 \mu \mathrm{m}$ of the different clouds. Column 4 contains the determined total masses of the clouds. The equivalent diameter $D(\mathrm{Col} .5)$ is an estimate of the cloud size, derived from the area of the clumps given by the ClumpFind algorithm. The mass of cloud a $(\mathrm{M} 0.25+0.012)$ is consistent with the results of Longmore et al. (2012).

The largest uncertainty in the mass determination arises from the determination of the cloud boundaries. Depending on the lowest contour level of the ClumpFind algorithm, the sizes of the clouds change slightly, thus including more or less diffuse emission at the boundaries of the clouds. For example, a change of the lowest contour level from $1.7 \mathrm{Jy} \mathrm{beam}^{-1}$ to $1.8 \mathrm{Jy}^{\mathrm{beam}^{-1}} \mathrm{de}$ creases the masses of the larger clouds by $1-5 \%$ and the smaller clouds by $\sim 10 \%$. The equivalent diameter decreases by $1-8 \%$ and we thus conclude that the size of the clouds is robust against small changes in the input level.

As mentioned in Sect. 2, the data reduction process was developed specifically for a better recovery of the extended emission. However, we cannot exclude that some uniform extended emission is still filtered out by the data reduction process. The masses we derive are therefore only lower limits of the real masses. Furthermore, the gas-to-dust-ratio in the central molecular zone may be lower than the standard value of 100 (determined at solar metallicity) due to the negative metallicity gradient in the disk (Balser et al. 2011). Since no metallicity measurements exist for the dust ridge region, we adopted the commonly used value of 100 for the gas-to-dust ratio, but we note that the total masses of the clouds may be lower. We estimate the uncertainty of the total cloud masses to be of the order of $\sim 20 \%$.

The total mass of the dust ridge adds up to $\sim 5 \times 10^{5} M_{\odot}$, which makes this region one of the most massive dust and gas reservoirs in the vicinity of the Galactic center. Therefore, probably an important site of the future star formation in the central molecular zone will develop in this region.

\subsection{Maser sources in the dust ridge}

Lis et al. (1994) conducted an observation of the cloud $\mathrm{M} 0.25+0.012$ at $22.2 \mathrm{GHz}$ with the VLA in the B/C hybrid configuration. They found a weak water maser near the peak of the submillimeter emission with an isotropic $22 \mathrm{GHz}$ line luminosity of $1.5 \times 10^{-6} L_{\odot}$. They deduced that this maser is most likely associated with a deeply embedded intermediate or low-mass young stellar object.

A recently conducted search for class II methanol masers at $6.7 \mathrm{GHz}$ (Caswell et al. 2010) showed the existence of three methanol masers in the dust ridge region. They are located in clouds $\mathrm{c}$ and e, as well as in the south-west of M0.25+0.012. At the positions of the first two methanol masers also water masers have been detected (Forster \& Caswell 1999; Valdettaro et al. 2001). The positions of the water and methanol masers are indicated with black and white crosses, respectively, in Fig. 2.

The existence of the methanol masers shows that high-mass star formation is taking place in parts of the dust ridge. However, besides the weak water maser, there were no other masers found in the most massive cloud M0.25+0.012.

\subsection{Radio sources in the dust ridge}

Figure 3 shows that the primary beams of the VLA observations cover only the dust ridge clouds a-d. Thus, we cannot make a statement about radio sources in clouds e and f from our observations. However, there are no $5 \mathrm{GHz}$ or $1.4 \mathrm{GHz}$ sources from the catalog of Becker et al. (1994) located in clouds e and f. In addition, radio emission is only detected towards the known $\mathrm{H}$ II region Sgr B1 in the south of clouds e and $\mathrm{f}$ in the $1.4 \mathrm{GHz}$ map of Yusef-Zadeh et al. (2004) and in VLA archival data at $4.8 \mathrm{GHz}$. We, therefore, exclude the existence of $\mathrm{H}$ II regions in the clouds e and $\mathrm{f}$.

A total of five radio sources are detected in our three $8.4 \mathrm{GHz}$ VLA fields. We fitted the sources in the primary beam corrected images with Gaussian models and determined their positions and angular sizes, as well as their peak and integrated flux densities. We calculated the spectral indices of the radio sources from the integrated flux densities at 8.4 and at $5 \mathrm{GHz}$ (from the catalog of Becker et al. 1994). Since Becker et al. (1994) list only the $1.4 \mathrm{GHz}$ peak flux densities of the counterparts in their catalog, integrated flux density values at $1.4 \mathrm{GHz}$ could not be included in the determination of the spectral index.

The image centered on POS1 contains three sources (labeled A, B, and C in Fig. 3) which are all offset from molecular cloud M0.25+0.012 to SWW, W and NE, respectively. Of these three sources, only the southernmost source (A) is associated with $870 \mu \mathrm{m}$ dust emission, as well as water and methanol masers (see Sect. 3.2 and Fig. 2). This source is also detected at 1.4 and $5 \mathrm{GHz}$ (Becker et al. 1994). Its spectral index is $\alpha \sim+0.3$, supporting the identification of this source as an $\mathrm{H}$ II region. 


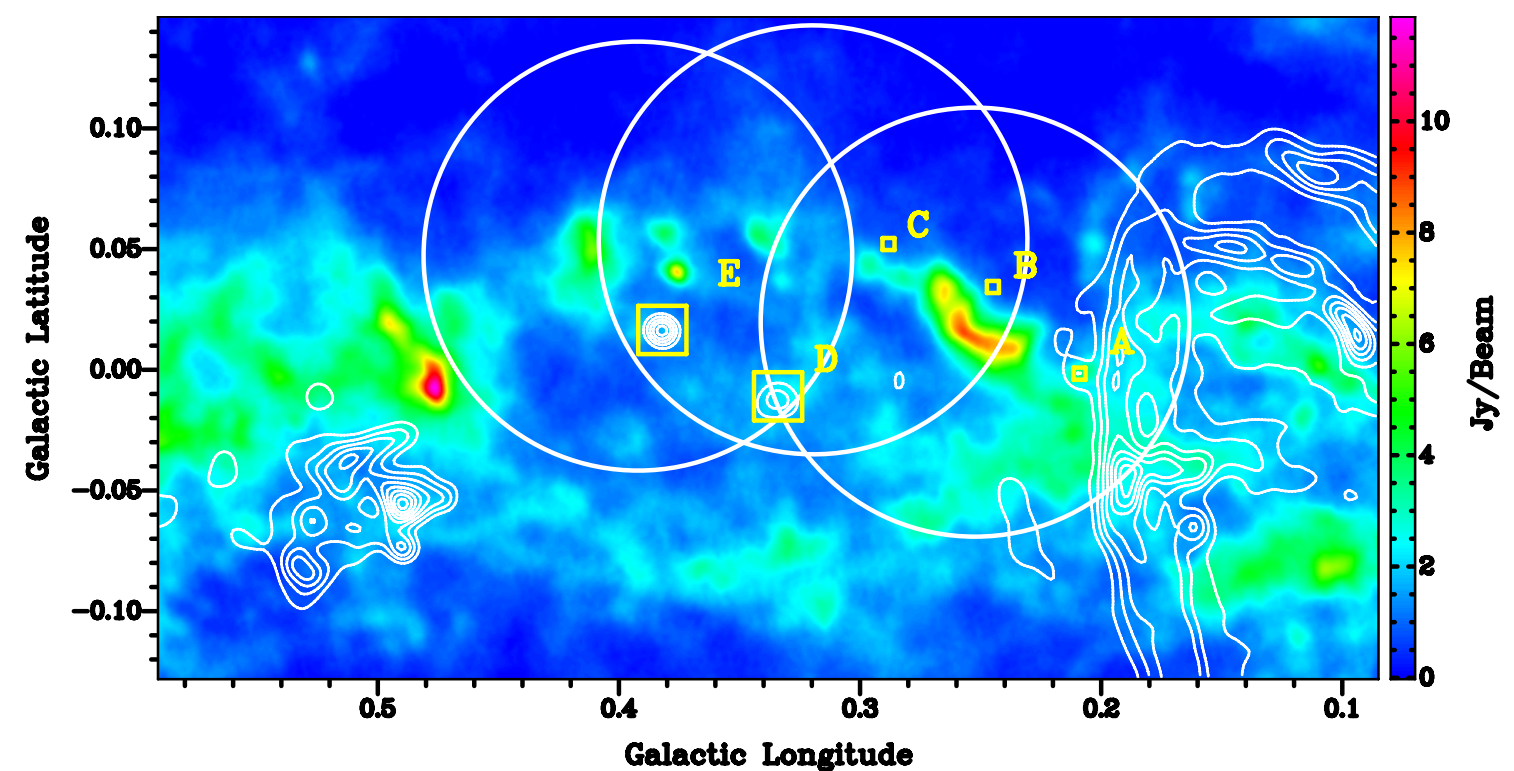

(a) Dust Ridge

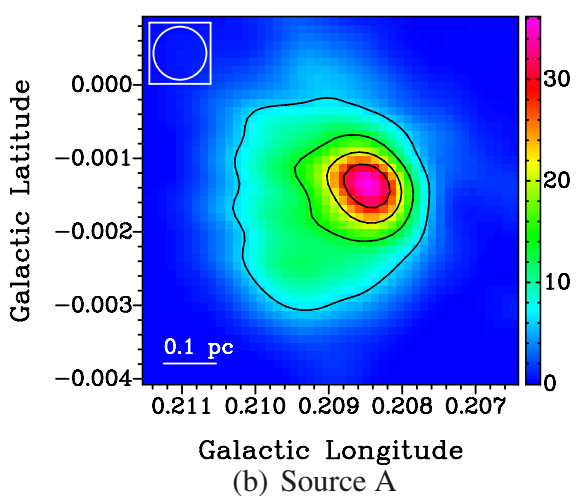

(b) Source A

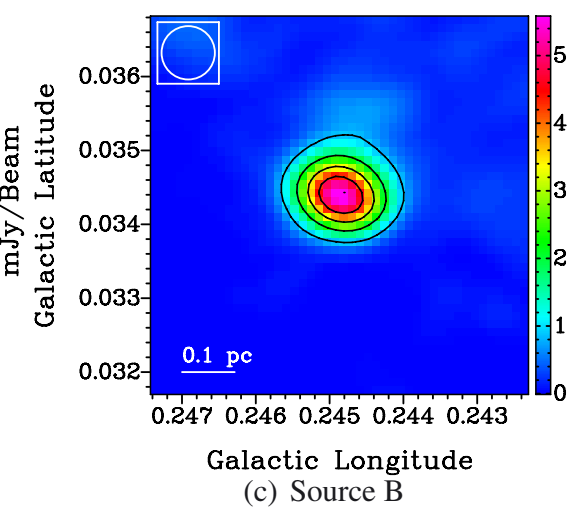

(c) Source B

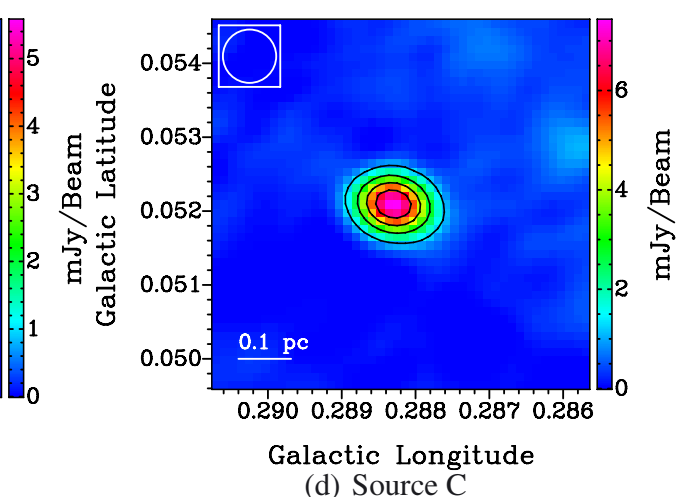

(d) Source C

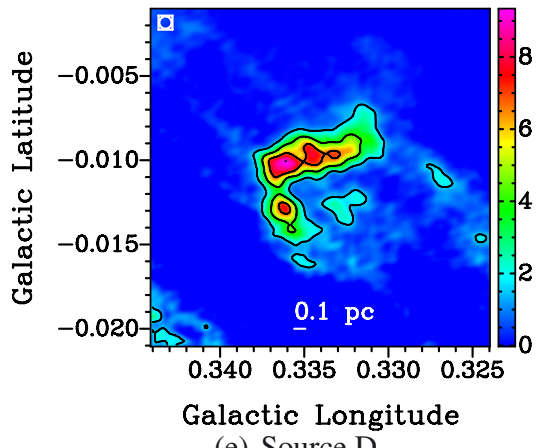

(e) Source D

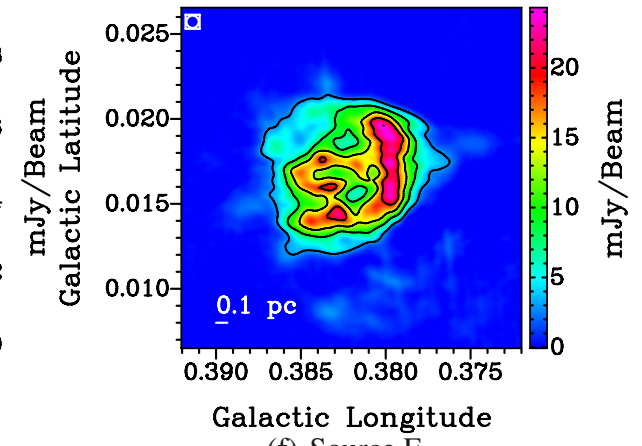

(f) Source E

Fig. 3. Panel a) $870 \mu \mathrm{m}$ dust emission toward the dust ridge (see Fig. 2) with overlaid contours of the $1.4 \mathrm{GHz}$ radio continuum emission (Yusef-Zadeh et al. 2004). Contour levels are 3\% to 10\%, in steps of $1 \%$. White circles show the VLA primary beam at $8.4 \mathrm{GHz}$ for the three pointings observed. Yellow boxes mark the positions and sizes of the radio continuum sources enlarged in panels b)-f). Panels b)-f) $8.4 \mathrm{GHz}$ radio continuum emission toward sources A-E. Contour levels are $20 \%$ to $100 \%$, in steps of $20 \%$ (1\% corresponds to $1.4 \sigma, 0.2 \sigma, 0.3 \sigma, 0.6 \sigma$, and $1.0 \sigma$ in Figs. 3b-f, respectively).

The second southernmost source (B) was also detected by Lis et al. (1994) who measured a peak flux density of $7.4 \mathrm{mJy}$ beam $^{-1}$ and an integrated flux density of $14.6 \mathrm{mJy}$ at 8.4 GHz. They doubted that this source has an extragalactic nature but instead assumed that they detected a compact $\mathrm{H}$ II region due to its relatively high flux density. The Gaussian fitting of our data results in a peak flux density of $5.56 \pm 0.58 \mathrm{mJy} \mathrm{beam}^{-1}$ and an integrated flux density of $9.27 \pm 1.42 \mathrm{mJy}$. Assuming similar uncertainties for the results of Lis et al. (1994), our results are consistent within $3 \sigma$.
The third source detected, C, is a small, nearly circularly shaped source which is also observable in the second field POS2. Its integrated flux density is even higher than the integrated flux density of source B. Source B and C are not detected in the $1.4 \mathrm{GHz}$ map of Yusef-Zadeh et al. (2004) or in the $5 \mathrm{GHz}$ catalog of Becker et al. (1994). We determined lower limits for the spectral index of -0.2 (source B) and 0 (source C), using the $5 \mathrm{GHz}$ detection limit of $9 \mathrm{mJy}$ as upper limit. The spectral indices suggest an interpretation of the two radio sources as H II regions. 
Table 3. Positions and integrated flux densities of the detected radio continuum sources.

\begin{tabular}{lccccccc}
\hline \hline Source & $\begin{array}{c}\text { RA (J2000) } \\
{[\mathrm{hh} \mathrm{mm} \mathrm{ss.ss]}]}\end{array}$ & $\begin{array}{c}\text { Dec (J2000) } \\
{\left[\mathrm{d}^{\prime \prime \prime \prime \prime \prime} .{ }^{\prime \prime \prime}\right]}\end{array}$ & $\begin{array}{c}S_{8.4 \mathrm{GHz}, \mathrm{Int} .} \\
{[\mathrm{mJy}]}\end{array}$ & $\begin{array}{c}S_{5 \mathrm{GHz}, \text { Int. }}{ }^{a} \\
{[\mathrm{mJy}]}\end{array}$ & $\alpha\left(S_{v} \propto v^{\alpha}\right)$ & $\begin{array}{c}\Theta_{R} \\
{\left[{ }^{\prime \prime}\right]}\end{array}$ & Comments \\
\hline A & 174607.35 & -284531.21 & $180 \pm 2$ & 154 & 0.3 & 5.1 & $S_{1.4 \mathrm{GHz}, \text { Peak }^{a} \sim 90 \mathrm{mJy} \mathrm{beam}^{-1}}$ \\
B & 174604.09 & -284233.17 & $9 \pm 1$ & $<9$ & $>-0.2$ & 1.8 & \\
C & 174606.15 & -283946.72 & $10 \pm 1$ & $<9$ & $>0$ & 1.8 & \\
D & 174627.56 & -283915.25 & $145 \pm 20$ & 134 & 0.2 & $14.1^{b}$ & $S_{1.4 \mathrm{GHz}, \text { Peak }} \sim 60 \mathrm{mJy} \mathrm{beam}^{-1}$ \\
E & 174628.93 & -283614.80 & $886 \pm 57$ & 1417 & -0.9 & 32.0 & $S_{1.4 \mathrm{GHz}, \text { Peak }} \sim 51 \mathrm{mJy} \mathrm{beam}^{-1}$ \\
\hline
\end{tabular}

Notes. ${ }^{(a)}$ Becker et al. (1994); ${ }^{(b)}$ at $5 \mathrm{GHz}$ (Becker et al. 1994).

Table 4. Physical parameters ${ }^{a}$ of the detected radio continuum sources.

\begin{tabular}{|c|c|c|c|c|c|c|}
\hline Source & $\begin{array}{c}S_{8.4 \mathrm{GHz}, \text { Int. }} \\
{[\mathrm{mJy}]}\end{array}$ & $\begin{array}{l}\Theta_{R} \\
{\left[{ }^{\prime \prime}\right]} \\
\end{array}$ & $\begin{array}{c}n_{\mathrm{e}} \\
{\left[\mathrm{cm}^{-3}\right]}\end{array}$ & $\begin{array}{c}E M \\
{\left[10^{5} \mathrm{~cm}^{-6} \mathrm{pc}\right]}\end{array}$ & $\begin{array}{l}M_{\mathrm{H}} \text { II } \\
{\left[M_{\odot}\right]} \\
\end{array}$ & $\begin{array}{c}\text { Spectral type } \\
\left(\text { single ZAMS star }{ }^{b}\right)\end{array}$ \\
\hline A & 180 & 5.1 & 2050 & 11.5 & 0.8 & O9-09.5 \\
\hline B & 9 & 1.8 & 2200 & 4.7 & 0.04 & B0-B0.5 \\
\hline $\mathrm{C}$ & 10 & 1.8 & 2330 & 5.3 & 0.04 & B0-B0.5 \\
\hline D & 145 & & & & & \\
\hline $\mathrm{E}$ & 886 & 32.0 & 290 & 1.4 & 28 & O6.5-07 \\
\hline
\end{tabular}

Notes. ${ }^{(a)}$ These values are calculated based on the assumption of a spherically symmetric H II region. Parameters of source D could not be determined, since the source is elongated. ${ }^{(b)}$ Obtained from Panagia (1973).

POS2 contains two more sources besides source $\mathrm{C}$, which lie close to the edge of the image in the area of higher noise values. Source D has a peculiar shape, it is not circular or nearly circular like the other sources, but is strongly extended in the north-west to south-east direction. Because of the elongation of the source together with its position in the field, one could assume that this source is spurious, but since the $1.4 \mathrm{GHz}$ map of Yusef-Zadeh et al. (2004) shows emission at this location and a $5 \mathrm{GHz}$ continuum source (Becker et al. 1994) coincides with this object, we conclude that this source is real. An explanation of the peculiar shape of this object could be the blending of several circular shaped sources. The spectral index of 0.2 supports the identification of this source as an $\mathrm{H}$ II region.

Source E is also observed in two fields (POS2, POS3) and has similar shapes in both fields. Of all the five sources, this source exhibits the strongest emission, with an integrated flux density of $0.89 \mathrm{Jy}$. This source was also detected in the $1.4 \mathrm{GHz}$ map of Yusef-Zadeh et al. (2004) and as a $5 \mathrm{GHz}$ continuum source by Becker et al. (1994) who identified it as an H II region. The spectral index is -0.9 , inconsistent with an $\mathrm{H}$ II region. However, the source is located in the higher-noise region at the edge of the field of view in both fields, which could explain a loss of emission for this source. Anderson et al. (2011) determined an integrated flux density of $1513 \pm 53 \mathrm{mJy}$ at $8.7 \mathrm{GHz}$ for this source, which results in a spectral index of 0.1 , again consistent with an $\mathrm{H}$ II region.

Although five sources are found in the three fields, no radio continuum sources at $8.4 \mathrm{GHz}$ could be detected within the dust ridge molecular clouds, indicating that no high-mass star formation is taking place, or that the high-mass star formation is in an early phase, where an $\mathrm{H}$ II region cannot yet be observed.

The positions (Cols. 2, 3) of the five sources (indicated with an upper-case letter, Col. 1), the integrated flux density values at 8.4 and $5 \mathrm{GHz}(\mathrm{Cols} .4,5)$, the spectral index from the integrated flux densities at 8.4 and $5 \mathrm{GHz}(\mathrm{Col} .6)$, and the angular radii $\Theta_{R}$ (Col. 7) are listed in Table 3.

Assuming that all these five sources have a Galactic origin and that they are all $\mathrm{H}$ II regions, excited by one zero-age main sequence star each, the spectral type of the stars that excite these regions can be estimated. This assumption is only questionable for source $\mathrm{C}$, since this source was not detected at other radio frequencies.

In order to determine the masses of the $\mathrm{H}$ II regions $M_{\mathrm{H} \text { II }}$, we calculate the electron density $n_{\mathrm{e}}$ and the emission measure $E M$ for the sources A, B, C, and E (see Appendix 4). Since the assumption of circular symmetric $\mathrm{H}$ II regions forms the basis of these calculations, they have not been conducted for source D. For the remaining sources, an electron temperature $T$ of $10^{4} \mathrm{~K}$ and a distance of $8.5 \mathrm{kpc}$ are assumed. The results of these calculations are listed in Table 4. Columns 2 and 3 show the measured integrated flux densities and the angular radii $\Theta_{R}$ at $8.4 \mathrm{GHz}$. Columns 4 and 5 contain the electron densities and the emission measure values. The masses of the $\mathrm{HII}$ regions are given in Col. 6. The spectral type of the stars is determined by comparing the number of Lyman continuum photons with the values from Table II of Panagia (1973) (shown in Col. 7). However, we cannot exclude that any of the detected sources are foreground objects which would mean that we overestimate their masses and the spectral types of the exciting stars.

The four sources show a wide range of $\mathrm{H}$ II masses from 0.04 to $28 M_{\odot}$, probably being excited by zero-age main sequence (ZAMS) stars with spectral types earlier than B0.5.

The most massive $\mathrm{H}$ II region (source E) needs for its excitation an $\mathrm{O} 6.5 \mathrm{~V}$ to $\mathrm{O} 7 \mathrm{~V}$ star. To put this source in perspective, let us compare it to the well studied archetypical compact H II region M42, the great nebula in Orion, the nearest site of active high-mass star formation (Genzel \& Stutzki 1989) at a distance of $414 \mathrm{pc}$ (Menten et al. 2007). The main exciting source of M42 is $\Theta_{1}$ Ori $C$, which has spectral type 07 V (Stahl et al. 2001). The left panel of Fig. 4 shows an image of the Orion nebula at $8.4 \mathrm{GHz}$, taken with the VLA in compact D-array (Shepherd et al. 2001). The integrated flux density of M 42 at $8.4 \mathrm{GHz}$ is $415 \mathrm{Jy}$ (Shepherd et al. 2001). The right panel shows source E at $8.4 \mathrm{GHz}$ for comparison on the same physical scale. Source E is only slightly larger than the Orion nebula. Scaling the flux density of the Orion nebula to the distance of source E yields a total 


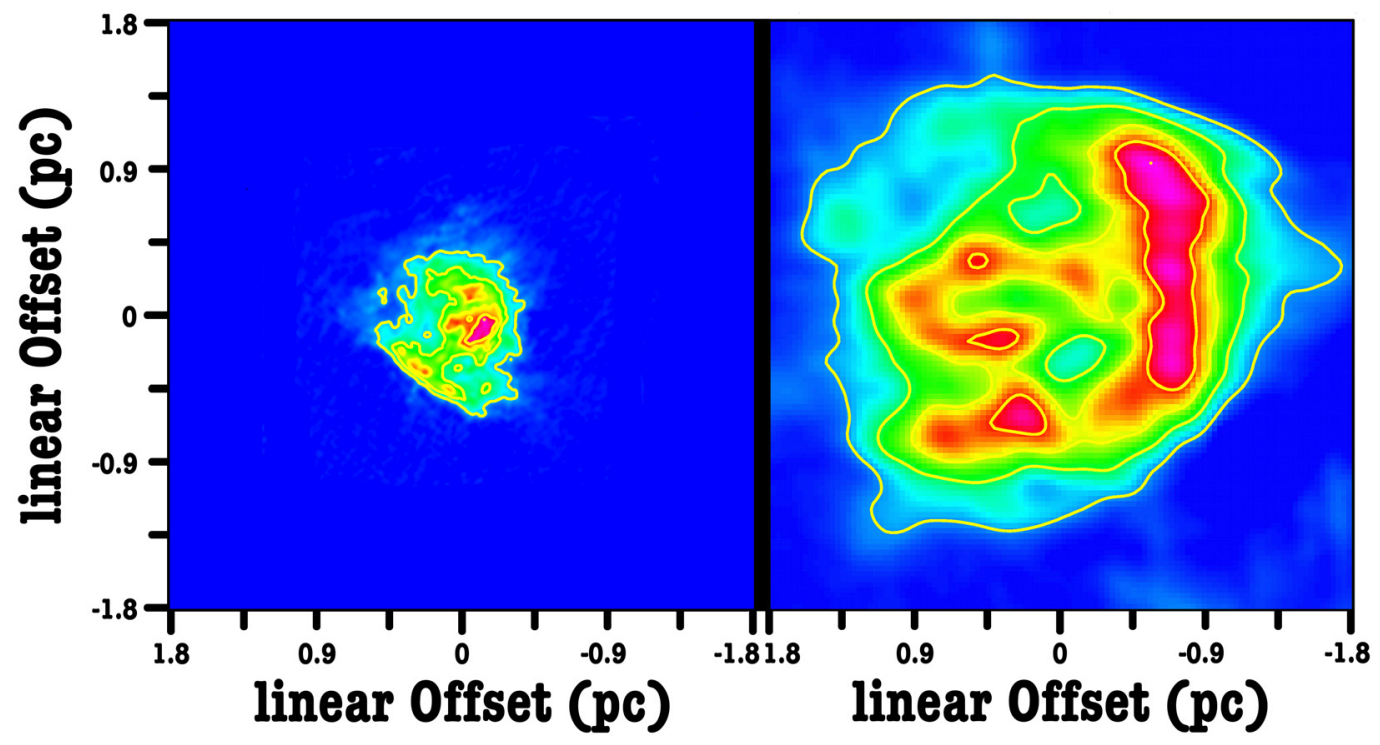

Fig. 4. Left: the Orion nebula (M42) at $8.4 \mathrm{GHz}$, as observed with the VLA in D-array (Shepherd et al. 2001). Right: source E at 8.4 GHz. Contour levels are $20 \%$ to $100 \%$, in steps of $20 \%$ in both panels (1\% corresponds to $1.4 \sigma$ and $1.0 \sigma$ in the left and the right panels, respectively).

flux density of $0.98 \mathrm{Jy}$, which is comparable to the integrated flux density of source E. Therefore, if we would move the Orion nebula to the Galactic center distance of $8.5 \mathrm{kpc}$, we would see an $\mathrm{H}$ II region similar to source E. While the massive pre-main sequence stars in Orion are easily detectable due to their closeness to the Sun, the large distance to source E prevents the detection of the exciting stars. Thus, the only sign of the massive star formation in source $\mathrm{E}$ is the strong radio continuum emission. Needless to say, it would be completely impossible to detect the emisison of the $\sim 2$ million year old, 2000 member Orion nebula cluster at optical wavelengths, if it had been placed in the Galactic center region, given the luminosity and the pervasive high extinction toward this region.

We conclude that our data show evidence for massive star formation in a more evolved state taking place at the periphery of the dust ridge region, but outside its molecular clouds. The $8.4 \mathrm{GHz} 3-\sigma$ sensitivity limit of $0.15-0.25 \mathrm{mJy}^{\text {beam }}{ }^{-1}$ in a 2". 6 beam can be translated to a Lyman continuum luminosity of $\sim 2.4-5 \times 10^{45} \mathrm{~s}^{-1}$ which corresponds to a single B0.5-B1 ZAMS star. Thus, we can exclude the existence of ZAMS star with spectral types earlier than B0.5 in the dust ridge clouds.

\subsection{Infrared emission in the dust ridge}

Observations of the dust ridge at infrared wavelengths give another possibility to find newly-born stars in the clouds, since these stars heat the surrounding dust which then reemits this energy at infrared wavelengths.

Figure 5 shows the dust ridge in a GLIMPSE false-color image. The contours display the distribution of the dust emission at $870 \mu \mathrm{m}$. The correspondence between the contours and the dark parts in the image in the northern part of the dust ridge is remarkable. The clouds in the southern part of the dust ridge are either not dense enough to absorb a large fraction of the diffuse infrared background or are located behind the material that emits the warm infrared background.

In addition, the infrared map shows emission at the positions of the three radio sources A, D, and E (marked with green crosses in Fig. 5). Since no maser emission is detected in the latter two sources, they might be in a more advanced stage of the star formation process.

\section{Conclusion}

Observation of the dust ridge at $870 \mu \mathrm{m}$ reveal several dust clouds ordered along a narrow ridge between the radio continuum sources G0.18-0.04 and Sgr B2. The temperature of the dust condensations is very low, between 15 and $22 \mathrm{~K}$, which indicates that if high-mass star formation is taking place in the clouds, it is in a very early stage. Mid-infrared observations of the dust ridge from the GLIMPSE survey show the dust ridge clouds in absorption in front of the diffuse infrared background. The agreement between the infrared dark clouds and the dust emission is remarkable.

The mass of the dust clouds are very high, ranging from $13000 M_{\odot}$ to over $150000 M_{\odot}$, making this region one of the most massive reservoirs of molecular material near the Galactic center. Due to the large masses and the low temperatures, it is possible that an active site of future star formation in the central molecular zone will develop in this region.

The detection of class II methanol masers in two of the dust ridge clouds is the only sign that massive stars are born in the dust ridge. Except a weak water maser, no sign of ongoing star formation is found in the most massive dust ridge cloud, M0.25+0.012.

Observations of the dust ridge region at $8.4 \mathrm{GHz}$ result in the detection of five radio continuum sources, which are probably all excited by young massive stars. However, the sources are found outside the massive dust clouds, in low column density regions, indicating that the star formation at these locations is in a more evolved state than in the dense clouds. The presence of stars with spectral types earlier than B0.5 in the dust ridge clouds is excluded by our observations.

M0.25+0.012 and cloud e are by far the most massive clouds in the dust ridge. Longmore et al. (2012) identified M0.25+0.012 as a possible precursor of a young massive cluster like the Arches cluster due to its position in the radius-mass plot. The radius and mass of cloud e are comparable to the values of M0.25+0.012. Assuming that cloud e is gravitationally bound, its position in the radius-mass plot would be very close to the position of M0.25+0.012 and this cloud could therefore also form a massive young cluster in the future. While M0.25+0.012 does not show any sign of star formation, cloud e contains a methanol 


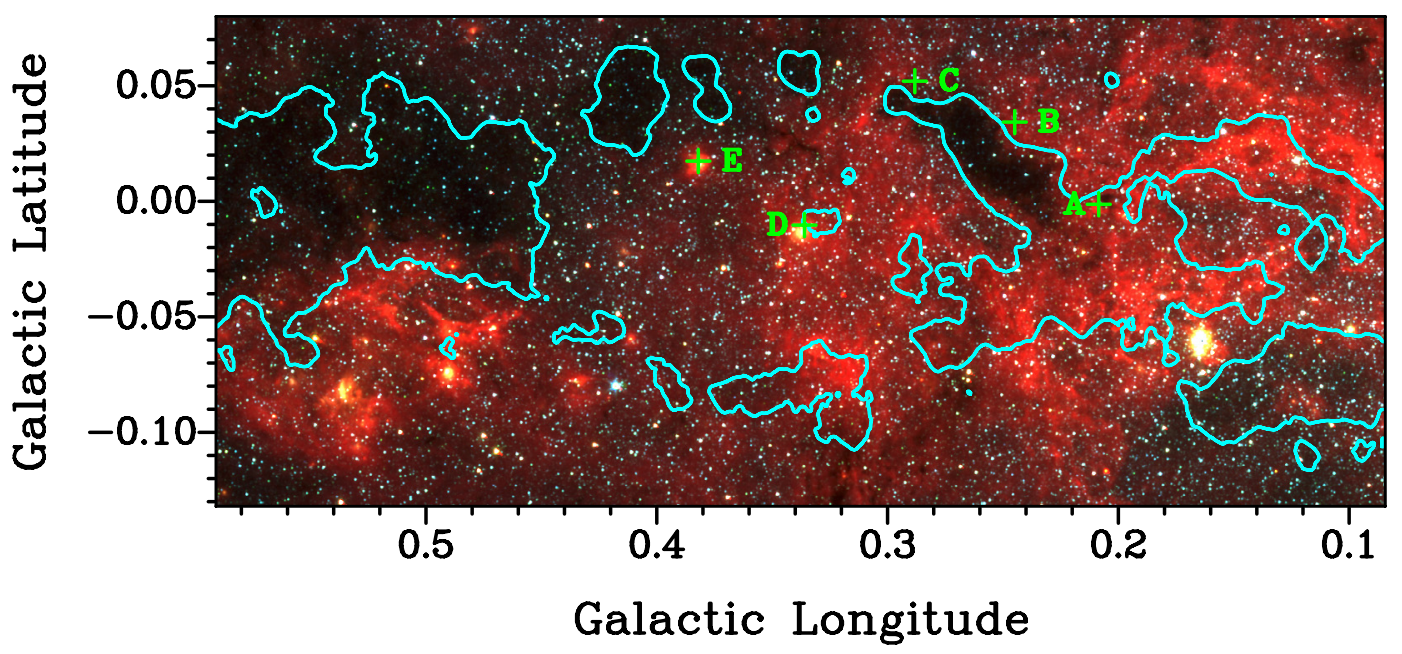

Fig. 5. Dust ridge clouds in absorption against the infrared background (GLIMPSE RGB image: blue $=3.6 \mu \mathrm{m}$, green $=4.5 \mu \mathrm{m}$, red $=8.0 \mu \mathrm{m}$ ). The contour shows dust emission at $20 \%$ of the peak, with $1 \%$ corresponding to $2 \sigma$. A strong correlation exists between the distribution of the dust emission and the shape of the infrared dark clouds. Green crosses and upper case letters mark the positions of the radio sources detected. Strong infrared emission is seen at the positions of the radio sources A, D, and E.

maser which indicates ongoing star formation in this cloud. We will use recent APEX spectral line observations of clouds a, d, and e in combination with data from the MALT90 survey to investigate the difference in chemistry between the clouds with and without signs of star formation and draw conclusions about the difference in the underlying physical conditions in the clouds.

Acknowledgements. The authors would like to thank Axel Weiss for his help in producing the image of the $870 \mu \mathrm{m}$ dust continuum emission of the central molecular zone (Fig. 1).

\section{Appendix A}

Following Panagia \& Walmsley (1978), the electron density $n_{\mathrm{e}}$ of a circular symmetric $\mathrm{H}$ II region can be determined with the formula:

$n_{\mathrm{e}}=311.3 \times\left[\frac{S}{\mathrm{Jy}}\right]^{0.5}\left[\frac{T}{10^{4} \mathrm{~K}}\right]^{0.25}\left[\frac{D}{\mathrm{kpc}}\right]^{-0.5} b(v, T)^{-0.5} \Theta_{R}^{-1.5} \mathrm{~cm}^{-3}$

with

$b(v, T)=1+0.3195 \log \left(\frac{T}{10^{4} \mathrm{~K}}\right)-0.213 \log \left(\frac{v}{1 \mathrm{GHz}}\right)$,

where $S$ denotes the flux densities, $T$ the temperatures, and $\Theta_{R}$ the angular radii of the sources. $D$ denotes the source distance and $v$ the frequency of the observation. Furthermore, the emission measure $E M$ can be estimated from the flux density $S$ and the angular radius $\Theta_{R}$ with the following formula, which can also be obtained from Panagia \& Walmsley (1978):

$E M=5.638 \times 10^{4}\left[\frac{S}{\mathrm{Jy}}\right]\left[\frac{T}{10^{4} \mathrm{~K}}\right] b(v, T) \Theta_{R}^{-2} \mathrm{~cm}^{-6} \mathrm{pc}$.

In addition, Tielens (2005) connects the emission measure with the number of Lyman continuum photons $N_{\text {Lyc }}$ that are necessary to produce this emission measure (a constant density nebula assumed):

$E M=1.6 \times 10^{6}\left[\frac{n_{\mathrm{e}}}{10^{3} \mathrm{~cm}^{-3}}\right]^{\frac{4}{3}}\left[\frac{N_{\mathrm{Lyc}}}{5 \times 10^{49} \text { photons s}}\right]^{\frac{1}{3}} \mathrm{~cm}^{-6} \mathrm{pc}$
From the number of Lyman continuum photons the spectral type of the new-born star can be determined (assuming the existence of only one star in the H II region) and, together with the electron density, the mass $M_{\mathrm{H} \text { II }}$ of the $\mathrm{H}$ II region can be calculated (Tielens 2005):

$M_{\mathrm{H} \text { II }} \approx 80 \times\left[\frac{n_{\mathrm{e}}}{10^{3} \mathrm{~cm}^{-3}}\right]^{-1}\left[\frac{N_{\mathrm{Lyc}}}{5 \times 10^{49} \text { photons s}^{-1}}\right] M_{\odot}$.

\section{References}

Anderson, L. D., Bania, T. M., Balser, D. S., \& Rood, R. T. 2011, ApJS, 194, 32 Balser, D. S., Rood, R. T., Bania, T. M., \& Anderson, L. D. 2011, ApJ, 738, 27 Becker, R. H., White, R. L., Helfand, D. J., \& Zoonematkermani, S. 1994, ApJS, 91, 347

Blaauw, A. 1964, ARA\&A, 2, 213

Caswell, J. L., Fuller, G. A., Green, J. A., et al. 2010, MNRAS, 404, 1029

Churchwell, E., Babler, B. L., Meade, M. R., et al. 2009, PASP, 121, 213

Forster, J. R., \& Caswell, J. L. 1999, A\&AS, 137, 43

Foster, J. B., Jackson, J. M., Barnes, P. J., et al. 2011, A\&AS, 197, 25

Genzel, R., \& Stutzki, J. 1989, ARA\&A, 27, 41

Ghez, A. M., Salim, S., Weinberg, N. N., et al. 2008, ApJ, 689, 1044

Güsten, R., Walmsley, C. M., \& Pauls, T. 1981, A\&A, 103, 197

Güsten, R., Nyman, L. À., Schilke, P., et al. 2006, A\&A, 454, L13

Hildebrand, R. H. 1983, Quart. J. RAS, 24, 267

Lis, D. C., \& Carlstrom, J. E. 1994, ApJ, 424, 189

Lis, D. C., \& Menten, K. M. 1998, ApJ, 507, 794

Lis, D. C., Menten, K. M., Serabyn, E., \& Zylka, R. 1994, ApJ, 423, L39

Lis, D. C., Li, Y., Dowell, C. D., \& Menten, K. M. 1999, in The Universe as Seen by ISO, eds. P. Cox, \& M. Kessler, ESA SP, 427, 627

Longmore, S. N., Rathborne, J., Bastian, N., et al. 2012, ApJ, 746, 117

Menten, K. M., Reid, M. J., Forbrich, J., \& Brunthaler, A. 2007, A\&A, 474, 515

Molinari, S., Swinyard, B., Bally, J., et al. 2010, PASP, 122, 314

Molinari, S., Bally, J., Noriega-Crespo, A., et al. 2011, ApJ, 735, L33

Panagia, N. 1973, ApJ, 78, 929

Panagia, N., \& Walmsley, C. M. 1978, A\&A, 70, 411

Schuller, F., Menten, K. M., Contreras, Y., et al. 2009, A\&A, 504, 415

Shepherd, D. S., Maddalena, R., \& MCMullin, J. P. 2001, in BAAS 33, AAS Meeting Abstracts, 134.03

Stahl, O., Wade, G., Petit, V., Stober, B., \& Schanne, L. 2008, A\&A, 487, 323

Tielens, A. G. G. M. 2005, The Physics and Chemistry of the Interstellar Medium (Cambridge Univ. Press)

Valdettaro, R., Palla, F., Brand, J., et al. 2001, A\&A, 368, 845

Williams, J. P., de Geus, E. J., \& Blitz, L. 1994, ApJ, 428, 693

Yusef-Zadeh, F., Hewitt, J. W., \& Cotton, W. 2004, ApJS, 155, 421 